\title{
Dynamic Model of a Giant Magnetostrictive Actuator System With Time Delay
}

\author{
Dongwei Li \\ School of Mechatronical Engineering \\ Beijing Institute of Technology \\ Beijing 100081, China \\ 295159081@qq.com
}

\author{
Dongwei Li, Zhaoshu Yang, Zhongbo He, Ce Rong \\ Vehicles and Electrical Engineering Department \\ Ordnance Engineering College \\ Shijiazhuang 050003, China
}

\author{
Weipeng Su \\ 76327 Troops \\ Chenzhou 423000, China
}

\begin{abstract}
Giant Magnetostrictive material (GMM), with the properties of fast response, great energy density and high Curie temperature, has potential to be applied to flow control, especially in designing of high speed servo valve working in severe circumstance. The Giant Magnetostrictive Actuator (GMA) is the component to produce tiny displacement precisely. It is the key component of high speed servo valve based on GMM. In order to establish a precise model of GMA, traditional linear time-invariant system model of the GMA can't describe the actuator displacement accurately. To predict the time delay and change law more effectively, the first order inertia and delay links are employed in the system model. The correctness and stability of the model are tested by a series of experiments. The traditional levy method is combined with Genetic Algorithm to identify the proposed model.
\end{abstract}

Keywords_-giant magnetostrictive actuator; linear system; inertia link; delay link

\section{INTRODUCTION}

The Giant Magnetostrictive Material (GMM), with the properties of fast response, great energy density and high Curie temperature, has potential uses in fields of flow control, active vibration control and precision machining, especially in designing of the high speed servo valve working in high temperature and high frequency circumstance. The Giant Magnetostrictive Actuator (GMA) is one of the most important applications of GMM, which could output tiny displacement in nanometers as well as a large force. This component can be applied on the advanced servo valve and precise flow control could be realized through the precise position control approached by GMA. As the bandwidth of GMM is very wide, this valve could be applied in high speed hydraulic control [1]. Modeling of GMA is very significant in the precise control of its displacement, while it is very hard to obtain a model because of its complex hysteresis and multifield coupling environment[2,3,4].

The modeling of the hysteresis of GMM divides into two approaches: physics-based and phenomenon-based hysteresis models. The physics-based model focus on the physical insight of hysteresis, so it can only be used in the systems with their substantial physics knowledge gained. One of the most well-known physics-based model is J-A model, which is derived from a series of macro magnetic formulation. This model describes magnetostriction as a process of domain wall motion[5,6]. The phenomenon-based hysteresis models deal with the hysteresis as an abstract mathematic description instead of providing a physical insight, so they are used more extensively than physics-based models. Recently researching works are focus on the nonlinear property study via modern numerical method such as neural network or genetic algorithms[7,8].

Whereas the nonlinear model of GMA is not used so extensively for the classical control theory is based on the linear time-invariant system, therefore the design of classical controllers such as PID controllers is prevented. Moreover, for real- time applications, the complexity of model may leading to tough hardware measurements without leading to any appreciable improvement in performance. Thus a simple and effective linear model of GMA is very significant to engineering application of GMM[9].

So far, literature on this field mainly focus on the modeling on GMA alone, instead of the whole GMA system including constant current sources [10,11]. Literature regard the whole GMA system as a first-order inertial system; Literature derived the third-order model of GMA system, but only the first-order approximation is identified. Whereas, in precise position control, the object is the whole GMA system, which is a series structure of GMA and constant current source. Because in practical applications, the digital input of constant current source is much easier to be controlled comparing to the current of actuator. The structure of GMA system can be described as Fig.1.

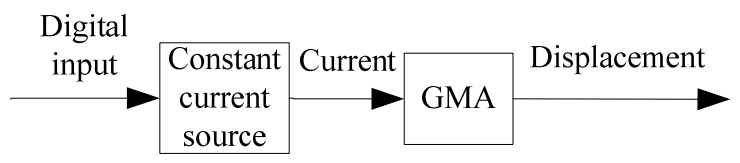

Fig.1 Structure of GMA system 


\section{MODELING DISPLACEMENT}

\section{A. Actuator Only}

Kinetic model of GMA is shown in Fig.1, $m_{M}, k_{M}$ and $c_{M}$ denote the quality, equivalent stiffness and damp of GMM rod. $m_{2}, k_{2}$ and $c_{2}$ denotes the equivalent quality, stiffness and damp of the load. Set the static Equilibrium point as origin point, the dynamic differential equation is derived as Eq.(1)

$$
m_{M} \ddot{x}_{1}+c_{M} \dot{x}_{1}+k_{M} x_{1}=F_{M}+f_{21}
$$

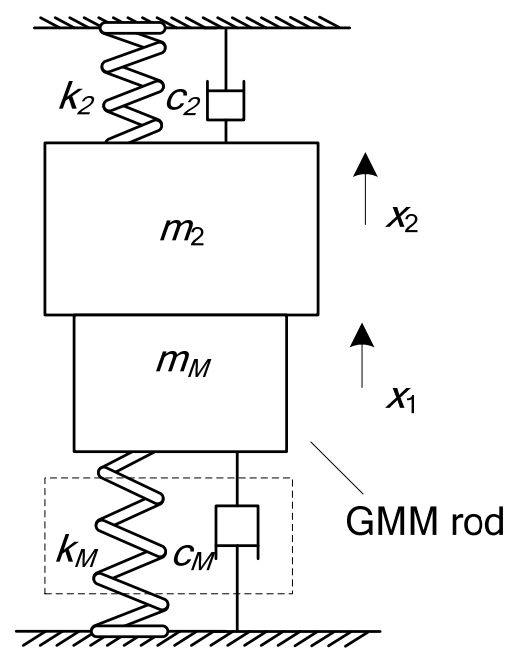

Fig.2 dynamic model of GMA

Where $F_{M}$ denotes the force produced by GMM rod, $f_{12}$ denotes the force of load to GMA. The dynamic differential equation of $m_{2}$ is

$$
m_{2} \ddot{x}_{2}+c_{2} \dot{x}_{2}+k_{2} x_{2}=f_{12}
$$

where, $f_{12}$ denotes the force from $m_{M}$ to $m_{2}$, i.e. $f_{12}=-f_{12}$; if the system is connected rigidly, $x_{1}=x_{2}$. Sum Eq. (1) and Eq.(2):

$$
\left(m_{2}+m_{M}\right) \ddot{x}_{2}+\left(c_{2}+c_{M}\right) \dot{x}_{2}+\left(k_{2}+k_{M}\right) x_{2}=F_{M}
$$

With the bias field exerted, the GMA could be considered working in the linear interval, so Eq.(3) could be expressed as

$$
\left(m_{2}+m_{M}\right) \ddot{x}_{2}+\left(c_{2}+c_{M}\right) \dot{x}_{2}+\left(k_{2}+k_{M}\right) x_{2}=k_{M} d_{33} N I
$$

Where, $d_{33}$ denotes the magnetic-mechanic coupling coefficient; $N$ denotes the turns of coil. In this way, the transfer function of GMA could be expressed as

$$
G_{1}(s)=\frac{X(s)}{I(s)}=\frac{K_{1}}{\left(m_{2}+m_{M}\right) s^{2}+\left(c_{2}+c_{M}\right) s+\left(k_{2}+k_{M}\right)}
$$

Where, $X(s)$ and $I_{s}(s)$ denotes the Laplace transform of $x_{2}$ and $I, K_{1}=k_{M} d_{33} N$.

\section{B. Whole actuating system}

GMA system is a series structure of constant current source and GMA, which is described as Fig.1. It is the component producing tiny displacement in GMM applications. With the extraordinary complex of its structure, the method of system identification should be introduced to approach the model of GMA system.

The constant current source could be regarded as a firstorder inertial part, the transfer function is:

$$
G_{0}(s)=\frac{K_{0}}{T_{0} s+1} e^{-L_{0} s}
$$

Then the transfer function of whole GMA system is

$$
G(s)=G_{0}(s) G_{1}(s)=\frac{K_{0} K_{1}}{\left(T_{0} s+1\right)\left(m s^{2}+c s+k\right)} e^{-\tau s}
$$

Where $m=m_{2}+m_{M}, c=c_{2}+c_{M}, k=k_{2}+k_{M}$. For GMA system, with the same input current and pre-stress, the system could be regarded as a time-invariant system. While because of the complexity of the GMA structure and constant current source structure, the parameters in Eq.(7) cannot be tested easily. In this paper, levy method is applied to identify the model, however, a model with time delay cannot be identified by traditional levy method. Thus the model should be transformed firstly. Eq.(7) could be transformed as

$$
G(s)=\frac{b_{0}}{1+a_{1} s+a_{2} s^{2}+a_{3} s^{3}} e^{-\tau s}
$$

The physical meanings of parameters in Eq.(8) can be expressed as $a_{1}=\left(T_{0} k+c\right) / k \quad, \quad a_{2}=\left(T_{0} c+m\right) / k$, $a_{3}=T_{0} m / k, b_{0}=K_{0} K_{1} / k$. Then, an estimate of $G(s)$ could be expressed as

$$
\hat{G}(s)=\frac{\hat{b}_{0}}{1+\hat{a}_{1} s+\hat{a}_{2} s^{2}+\hat{a}_{3} s^{3}} e^{-\hat{\tau} s}
$$

Obviously, $\hat{b}_{0}$ denotes the open-loop gain of system, denoting $\hat{a}=\left[\hat{a}_{1}, \hat{a}_{2}, \hat{a}_{3}\right]^{T}$, the problem is attributed to the estimate of $\hat{a}$ and $\hat{\tau}$. Noting that amplitude-frequency characteristic will not be influenced by time delay element, let

$$
F(w)=\hat{G}(-j w) \hat{G}(j w)=\frac{d_{0}}{1+c_{1} w^{2}+c_{2} w^{4}+c_{3} w^{6}}
$$

Obviously $d_{0}=b_{0}^{2}$, denoting $C(w)=1+c_{1} w^{2}+c_{2} w^{4}+c_{3} w^{6}$, then, the levy method is introduced to identify $C(w)$. The error function is defined as 


$$
\tilde{\varepsilon}_{i}=F\left(w_{i}\right) C\left(w_{i}\right)-d_{0}
$$

And the performance function is set as

$$
J=\sum_{i=1}^{N}\left(\left|M\left(w_{i}\right)\right|^{2} C\left(w_{i}\right)-d_{0}\right)^{2}
$$

Where $M\left(w_{i}\right)$ and $t_{d}\left(w_{i}\right)$ denote the amplitude ratio and time delay between input and output signals respectively. The optimum solution of $J$ satisfy the following conditions ;

$$
\frac{\partial J}{\partial c_{j}}=2 \sum_{i=1}^{N} \tilde{\varepsilon}_{i} \times \frac{\partial \tilde{\varepsilon}_{i}}{\partial C\left(w_{i}\right)} \times \frac{\partial C\left(w_{i}\right)}{\partial c_{j}}=0, j=1,2,3
$$

Noting that $F(w)=\hat{G}(j w) \hat{G}(-j w)$, thus real part of $F(w)$ equals to $\left|M\left(w_{i}\right)\right|^{2}$, while the imaginary part equals to 0 . Two variables are introduced:

$$
\left\{\begin{array}{l}
S_{h}=\sum_{i=1}^{N} w_{i}{ }^{h} M\left(w_{i}\right)^{2} \\
U_{h}=\sum_{i=1}^{N} w_{i}{ }^{h} M\left(w_{i}\right)^{4}
\end{array}\right.
$$

Then Eq.13 could be expressed as

$$
\left(\begin{array}{ccc}
U_{4} & U_{6} & U_{8} \\
U_{6} & U_{8} & U_{10} \\
U_{8} & U_{10} & U_{12}
\end{array}\right)\left(\begin{array}{l}
c_{1} \\
c_{2} \\
C_{3}
\end{array}\right)=\left(\begin{array}{cc}
S_{2} & U_{2} \\
S_{4} & U_{4} \\
S_{6} & U_{6}
\end{array}\right)\left(\begin{array}{c}
d_{0} \\
-1
\end{array}\right)
$$

Excited by a series of sine signals with different frequencies, the input and output signals are acquired, filtered with a cutoff frequency of $2 \mathrm{kHz}, M\left(w_{i}\right)$ and $t_{d}\left(w_{i}\right)$ varies with exciting frequencies is obtained. The result of identification is shown as Eq.(16)

$$
F(w)=\frac{3.3451 \times 10^{-9}}{1+3.0052 \times 10^{-7} w^{2}-2.4459 \times 10^{-14} w^{4}+5.8812 \times 10^{-22} w^{6}}
$$

Solving the characteristic equation of $F(w)$, six characteristic roots are obtained, learning from Literature, characteristic roots of $G(j w)$ could be expressed as

$$
p_{k}=j w_{k}^{c}
$$

According to Routh stability criterion, to guarantee the stability of system, only the characteristic root with a negative real part could be selected. Thus, the characteristic roots of $G(j w)$ are selected as

$$
\left\{\begin{array}{l}
p_{1}=-1642.3 \\
p_{2}=-1217.7+4860.6 j \\
p_{3}=-1217.7+4860.6 j
\end{array}\right.
$$

Substituted into characteristic equation of $G(j w)$

$$
A(s)=1+\widehat{a}_{1} s+\widehat{a}_{2} s^{2}+\widehat{a}_{3} s^{3}=0
$$

$A(s)$ is finally obtained as

$$
\begin{aligned}
A(s) & =1+7.0590 \times 10^{-4} s+9.8888 \times 10^{-8} s^{2} \\
& +2.4251 \times 10^{-11} s^{3}
\end{aligned}
$$

Tested from experiment, the open-loop gain $b_{0}=G(0)=5.78 \times 10^{-5}$. Then an estimate of $G(s)$ is expressed as

$$
\hat{G}(s)=\frac{5.78 \times 10^{-5} e^{-\bar{\tau} s}}{1+7.059 \times 10^{-4} s+9.8888 \times 10^{-8} s^{2}+2.4251 \times 10^{-11} s^{3}}
$$

Denoting $\widehat{G}(s)=\hat{H}(s) e^{-\hat{\tau} s}$, learning from Literature,

$$
\widehat{\tau}\left(w_{i}\right)=t_{d}\left(w_{i}\right)-\operatorname{Arg} \hat{H}\left(j w_{i}\right) / \omega_{i}, i=1,2,3 \cdots m
$$

In this paper, the Delay Parameter $\tau$ is estimated via parameter optimization. The performance function is selected as

$$
J_{1}=\sum_{i=1}^{N}\left(G\left(j w_{i}\right)-H\left(j w_{i}\right) e^{-\bar{\tau} j w_{i}}\right)^{2}
$$

Substituted $\widehat{\tau}\left(w_{i}\right)$ of each frequency point into Eq.(23), The distribution of $J_{1}$ in frequency domain is shown as Fig.3. With a multi-peak distribution appeared, the global search capability of the optimization algorithm should be considered avoiding to get a local optimum solution only.

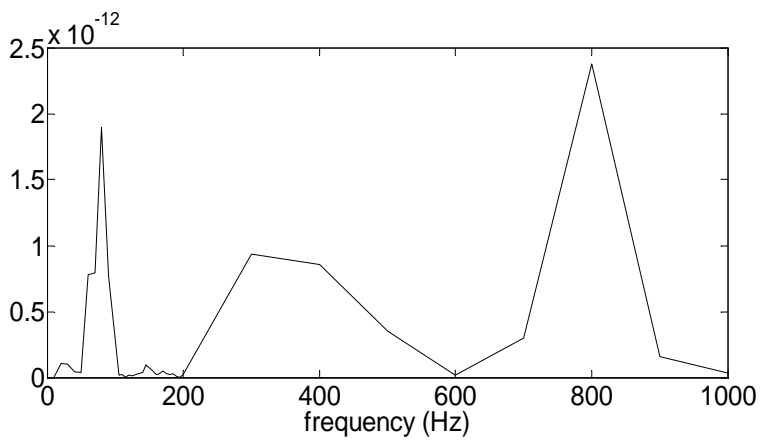

Fig.3 Distribution of $J_{1}$ with frequency 
Thus, genetic algorithm is applied in searching the optimum solution of $\tau$. The fitness function is selected as $J_{1}$ directly. Using algorithm cross:

$$
\left\{\begin{array}{l}
x_{v}^{t+1}=\alpha x_{w}^{t}+(1-\alpha) x_{v}^{t} \\
x_{w}^{t+1}=\alpha x_{v}^{t}+(1-\alpha) x_{w}^{t}
\end{array}\right.
$$

$x_{v}^{t}$ and $x_{w}^{t}$ denote the chromosome to be crossed, $x_{v}^{t+1}$ and $x_{w}^{t+1}$ denote the next generation. $\alpha=0.5$, mutation probability $p_{m}=0.0005$. The size of population is 40 , the range of delay parameter is $0 \leq \tau \leq 0.0023$ after 51 iterations, the identification is $\tau=0.00084 \mathrm{~s}$. Thus, the transfer function of GMA system is

$$
\hat{G}(s)=\frac{5.78 \times 10^{-5} \mathrm{e}^{-8.4 \times 10^{-4} s}}{1+7.059 \times 10^{-4} s+9.8888 \times 10^{-8} s^{2}+2.4251 \times 10^{-11} s^{3}}
$$

\section{MODEL VALIDATION}

In order to valid the proposed model, a series of open-loop experiments are conducted. The setup of experiment is shown as Fig.4.



Fig.4 Setup of open-loop experiment

The main devices of experiment include: Picoscope2203 acts as signal generator and data acquisition; displacement sensor is selected as CWY-DO-500, it is an eddy current sensor produced by Yangzhou Radio Plant; the amplifier is selected as YE5874 which is also produced by Yangzhou Radio Plant, the feedback method of amplifier could be altered between voltage mode and current mocde, as current feedback method selected, the amplifier acts as a constant current source.

Considering that the frequency components of square wave is very wide, allowing to excite most of the frequency components of GMA system; and in practical use, the driving signal of servo valve is PWM signal, thus a series of square waves with different pulse width and duty circle are chosen as the exciting signals, the corresponding output displacements are tested. With the excitation, the simulated outputs of proposed model are obtained in SIMULINK. Compared with the real test displacements, the effectiveness of proposed model is valided.

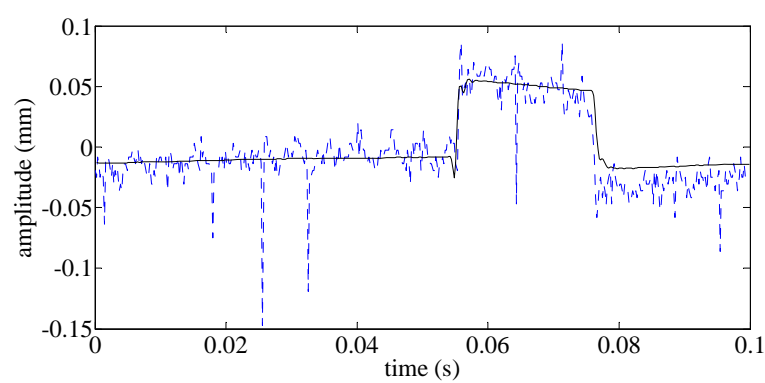

(a) Pulse width 0.1 s, duty cycle $20 \%$

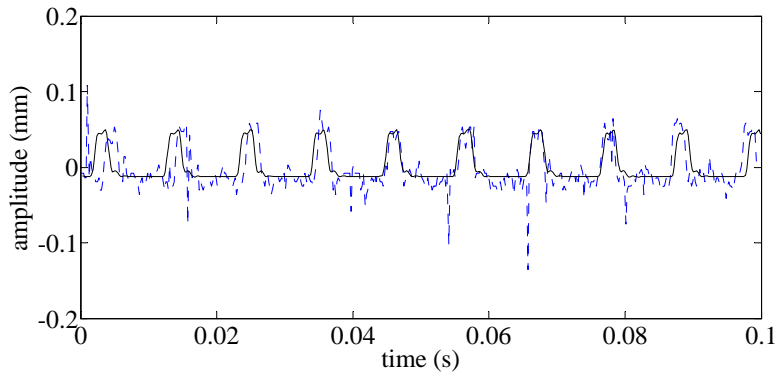

(b) Pulse width $0.01 \mathrm{~s}$, duty cycle $20 \%$

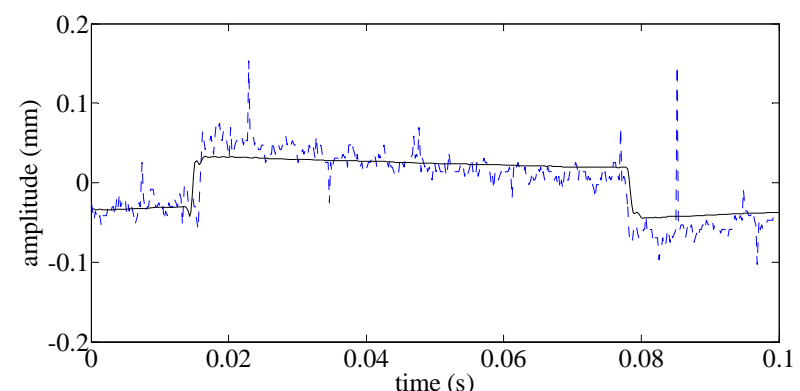

(c) Pulse width 0.1 s, duty cycle $60 \%$

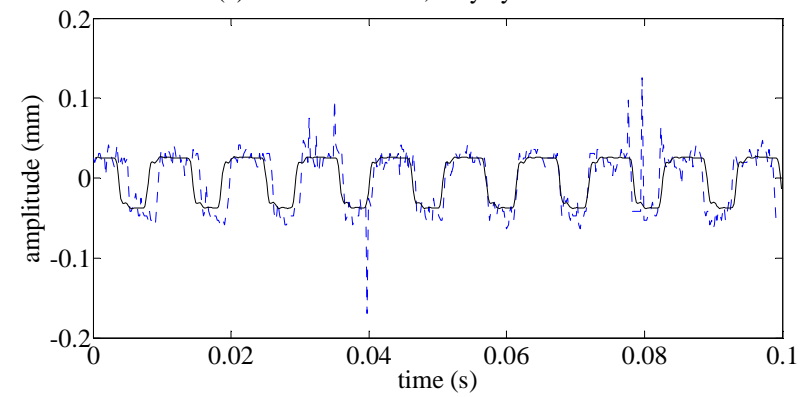

(d) Pulse width $0.01 \mathrm{~s}$, duty cycle $60 \%$

Fig.5. comparison of simulating and tested outputs excited by different frequencies and duty cycles

\section{CONCLUSION}

In this paper, the linear model of GMA system is derived, the traditional levy method is combined with Genetic Algorithm to identify the proposed model, and its effectiveness is tested by a series of open-loop experiments. traditional linear time-invariant system model of the GMA can't describe the actuator displacement accurately. First order inertia and delay links are employed to predict the time delay and changing law more effectively. The correctness of the model are verified by a series of experiments. 


\section{Acknowledgment}

This paper was supported by National Natural Science Foundation of China under Grant No. 51275525.

\section{References}

[1] S. Karunanidhi, M. Singaperumal, Design, analysis and simulation of magnetostrictive actuator and its application to high dynamic servo valve, Sensors and Actuators A: Physical. 157(2010)185-197.

[2] K.W. Zhang, Z.-Y. Cheng, et al, Magnetostrictive resonators as sensors and actuators, Sensors and Actuators A: Physical. (2013), http://dx,doi.org/10.1016/j.sna.2012.12.013.

[3] A.G. Olabi, A. Grunwald, Design and application of magnetostrictive materials, Materials and Design 29(2008)469-483.

[4] F. Braghin, S. Cinquemani, et al, A low frequency magnetostrictive inertial actuator for vibration control, Sensors and Actuators A: Physical 180(2012)67-74.
[5] D.C. Jiles, Modeling the effect of eddy current losses on frequency dependent hysteresis in electrically conducting media, IEEE Transactions on Magnetics 30(6)(1994)4326-4328.

[6] Z.J. Chen, D.C. Jiles, Modeling of reversible domain wall motion under the action of magnetic field and localized defects, IEEE transaction on magnetics 29(6)(1993)2554-2556.

[7] Y. Sun, B. Wang, et al, hysteresis compensation control algorithm for the giant magnetostrictive actuators, IEEE international conference on mechatronics and automation (2006) 2139-2143.

[8] S. Cao, B. Wang, et al, Modeling dynamic hysteresis for giant magnetostrictive actuators, IEEE transactions on magnetics 42(4)( 2006)911-914.

[9] F. Braghin, S. Cinquemani, F. Resta. A Model of Magnetostrictive Actuators for Active Vibration Control. Sensors and Actuators A 165(2011)342-350.

[10] D.F. Chen, L. Shu, et al, Modeling and control simulation of giant magnetostrictive actuator. Chinese Journal of Mechanical Engineering 2007, 43(8)12-16.

[11] Y. Sun, B.W. Wang, et al, Dynamic Linearity Model and Experiment for a Giant Magnetostrictive Actuator. Proceedings of the CSEE, 27(18)(2007) 96-100. 